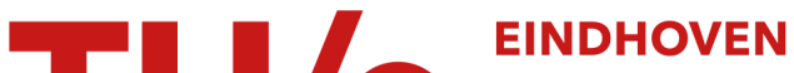 \\ UNIVERSITY OF \\ TECHNOLOGY
}

\section{Robust attenuation of direct-drive robot-tip vibrations}

Citation for published version (APA):

Kostic, D., Jager, de, A. G., \& Steinbuch, M. (2002). Robust attenuation of direct-drive robot-tip vibrations. In Proceedings of the International Conference on Intelligent Robots and Systems (2002 IEEE/RSJ), October 2002, Lausanne, Switzerland (pp. 2206-2212). Institute of Electrical and Electronics Engineers.

https://doi.org/10.1109/IRDS.2002.1041595

DOI:

10.1109/IRDS.2002.1041595

Document status and date:

Published: 01/01/2002

\section{Document Version:}

Publisher's PDF, also known as Version of Record (includes final page, issue and volume numbers)

\section{Please check the document version of this publication:}

- A submitted manuscript is the version of the article upon submission and before peer-review. There can be important differences between the submitted version and the official published version of record. People interested in the research are advised to contact the author for the final version of the publication, or visit the $\mathrm{DOI}$ to the publisher's website.

- The final author version and the galley proof are versions of the publication after peer review.

- The final published version features the final layout of the paper including the volume, issue and page numbers.

Link to publication

\section{General rights}

Copyright and moral rights for the publications made accessible in the public portal are retained by the authors and/or other copyright owners and it is a condition of accessing publications that users recognise and abide by the legal requirements associated with these rights.

- Users may download and print one copy of any publication from the public portal for the purpose of private study or research.

- You may not further distribute the material or use it for any profit-making activity or commercial gain

- You may freely distribute the URL identifying the publication in the public portal.

If the publication is distributed under the terms of Article $25 \mathrm{fa}$ of the Dutch Copyright Act, indicated by the "Taverne" license above, please follow below link for the End User Agreement:

www.tue.nl/taverne

Take down policy

If you believe that this document breaches copyright please contact us at:

openaccess@tue.nl

providing details and we will investigate your claim. 
Proceedings of the 2002 IEEE/RSJ

Intl. Conference on intelligent Robots and Systems

EPFL, Lausanne, Switzerland • October 2002

\title{
Robust Attenuation of Direct-Drive Robot-Tip Vibrations
}

\author{
Dragan Kostić ${ }^{1}$, Bram de Jager ${ }^{2}$, Maarten Steinbuch ${ }^{3}$ \\ Department of Mechanical Engineering, Eindhoven University of Technology, Eindhoven, The Netherlands \\ 'D.Kostic@tue.nl, ${ }^{2}$ A.G.de.Jager@wfw.wtb.tue.nl, ${ }^{3}$ M.Steinbuch@tue.nl
}

\begin{abstract}
This paper suggests and employs a method for reducing vibrations at the tip of a robot. The vibrations, caused by structural flexibility, are detected using accelerometers. The robot control system consists of two complementary sub-systems: a nominal motion controller and $a$ vibration compensator. The former one realizes joint motions in accordance with a prescribed tip trajectory. The latter one robustly attenuates oscillations at the tip that are due to structural flexibility. The benefits of this set-up are experimentally verified for a spatial directdrive robot with 3 revolute degrees of freedom.
\end{abstract}

\section{Introduction}

Elastic effects in robotic systems are under permanent research during the last two decades [1-11]. They became especially important when using lightweight materials for robot constructions that may enable faster movements of robots with the same actuators applied. As the stiffness of lightweight constructions is in general not sufficient to avoid their elastic bending, quick movements may cause undesirable vibrations of the robot end-effector. Such vibrations deteriorate the accuracy of trajectory tracking.

A part of the research on robot flexibility is devoted to dynamic modeling of elastic effects [1-6]. The models can be used for simulation and control purposes. There are two classes of flexibility in robots: lumped elasticity in the transmission of torque $[1,2]$, and distributed link flexibility [3-6]. The former one arises due to the use of elastic transmission elements, e.g., harmonic drives, belts, or long shafts. The latter one comes as a result of using lightweight materials and constructions to reduce mass and inertia of the links. Such materials and constructions increase the link compliance, which leads to structural vibrations at low frequencies and of significant amplitude.

Dynamic models incorporating flexible behavior can be used for design of model-based controllers, which should counteract elastic effects [6-11]. The models implement feedforward control actions applied in addition to suitable linear/nonlinear feedback [6]. Apart from simulation studies, experimental results in application of vibration compensators are rarely presented in the literature. The most frequently encountered are single [7,8] or two-link planar flexible robot arms [9]. There are only a few references treating more complex spatial robot configurations, e.g., [10,11]. Possible reasons are the complexity of measuring equipment and a high dimension of the controllers, as advanced vibration control requires feedback from deformation variables in addition to nominal motion coordinates. Deformation variables represent link/joint deflections measured using strain gauges, accelerometers, visual systems, etc.

In this paper we propose an efficient control strategy for attenuating tip vibrations, applicable to general spatial robot configurations. Accelerometers attached at the robot tip are used as vibration sensors. The number of translational and angular directions along which reduction of vibrations is possible equals the number of degrees-of-freedom (d.o.f.). The dynamics along each direction is experimentally identified and used in the design of the vibration compensator. Benefits of the compensator are experimentally verified on a spatial robot with 3 revolute d.o.f. This robot belongs to the class of direct drive robots, which is usually recognized as very challenging for accurate control because of highly nonlinear and coupled dynamics. We achieved robust attenuation of vibrations arising at the tip, which encourages us that the suggested strategy is practically applicable to any mechanical system having an open kinematic chain. The key contributions of our approach are: $(i)$ time-efficient identification of elastic effects and their incorporation into the model of rigid-body dynamics, (ii) use of the resulting non-linear dynamic model to simplify the problem of vibration compensation to an ordinary regulation problem, solvable using a well-developed linear theory, and (iii) robust compensation of tip vibrations.

In the next section we include elastic effects into a rigidbody model of robot dynamics. The extended model is used for design of a vibration compensator. Section 3 describes our robotic set-up and analyses sources and effects of the robot flexibility. Identification of the elastodynamics, as well as design of a vibration compensator, are presented in Section 4. Section 5 shows experimental results that validate our approach. Conclusions are given at the end.

\section{Compensating for Flexible Dynamics}

The standard model of rigid-body robot dynamics [12]

$$
\boldsymbol{\tau}=\mathbf{H}(\mathbf{q}) \ddot{\mathbf{q}}+\mathbf{h}(\mathbf{q}, \dot{\mathbf{q}})
$$

is considered. Here, $\mathbf{q}, \dot{\mathbf{q}}$, and $\ddot{\mathbf{q}}$ are $n \times 1$ vectors of 
joint motions, velocities and accelerations ( $n$ is the number of d.o.f.), $\tau$ an $n \times 1$ vector of generalized joint forces, $\mathbf{H}$ an $n \times n$ inertia matrix, and $\mathbf{h}$ an $n \times 1$ vector of Coriolis/centripetal, gravitational and friction forces. When structural flexibility is negligible, equation (1) defines joint torques that correspond to a certain location (position and orientation) of the end-effector. This location is mapped to displacements in the joints via an inverse kinematics mapping [13]. When flexible dynamics is present, the location of the end-effector deviates from its rigid-body location, as illustrated in Fig. 1.

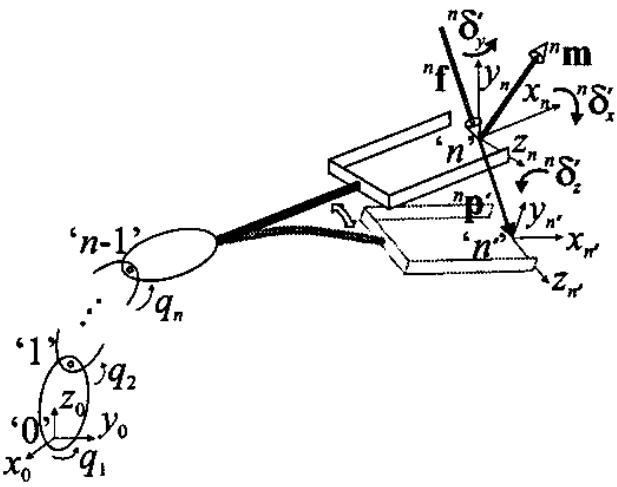

Figure 1: Elastic displacement of robot tip from its rigid-body location

We assume that dominant flexibility is due to elasticity in the most distal link. This is an important case in practical applications - think of robots used in space missions or machines for concrete pumping in civil engineering (boom pumps). Reference [10] suggests that vibrations at the tip, caused by elasticity, are the result of two factors: an equivalent elastic force ${ }^{n} \mathbf{f}$ and an equivalent elastic torque ${ }^{n} \mathbf{m}$. Both factors are indicated in Fig. 1, where they are referred to the tip coordinate frame ' $n$ '. They can be integrated into the model (1) using the well-known description of dynamic interaction between the end-effector and environment [13]:

$$
\begin{aligned}
\boldsymbol{\tau} & =\mathbf{H}(\mathbf{q}) \ddot{\mathbf{q}}+\mathbf{h}(\mathbf{q}, \dot{\mathbf{q}})+ \\
& +\mathbf{J}^{T}(\mathbf{q})\left[\begin{array}{cc}
{ }_{n}^{0} \mathbf{R}(\mathbf{q}) & \mathbf{0}_{3 \times 3} \\
\mathbf{S}(\mathbf{p}){ }_{n}^{0} \mathbf{R}(\mathbf{q}) & { }_{n}^{0} \mathbf{R}(\mathbf{q})
\end{array}\right]\left[\begin{array}{c}
{ }^{n} \mathbf{f}\left({ }^{n} \mathbf{p}\right) \\
{ }^{n} \mathbf{m}\left({ }^{n} \boldsymbol{\delta}^{\prime}\right)
\end{array}\right],
\end{aligned}
$$

where $\mathbf{J}(\mathbf{q})$ denotes a $6 \times n$ manipulator geometric Jacobian reffered to the base (inertial) frame ' 0 ', ${ }_{n}^{0} \mathbf{R}(\mathbf{q})$ is an orthogonal matrix mapping orientation of ' $n$ ' to ' 0 ', $\mathbf{p}$ is a $3 \times 1$ vector of tip Cartesian coordinates referred to ' 0 ', and the skew-symmetric matrix operator $\mathbf{S}$ is defined by:

$$
\mathbf{S}(\mathbf{p})=\left[\begin{array}{ccc}
0 & -p_{z} & p_{y} \\
p_{z} & 0 & -p_{x} \\
-p_{y} & p_{x} & 0
\end{array}\right]
$$

Variables ${ }^{n} \mathbf{p}^{\prime}$ and ${ }^{n} \boldsymbol{\delta}^{\prime}$ denote $3 \times 1$ vectors of translatio- nal and angular vibration coordinates, respectively, reffered to ' $n$ '. They represent displacement of the tip frame from its rigid-body location, as shown in Fig. 1.

The last term in (2) points out how to compensate for the manipulator flexibility. It indicates that control of reference joint motions, which we denote with $\tau_{q}$, has to be corrected with a term counteracting the effects of ${ }^{n} \mathbf{f}$ and ${ }^{n} \mathbf{m}$. This term we denote with $\boldsymbol{\tau}_{v}$. Let us adopt the computed torque control [13] for tracking a joint reference $\mathbf{q}_{r}(t)$. If we define the tacking error as

$$
\mathbf{e}(t)=\mathbf{q}_{r}(t)-\mathbf{q}(t),
$$

then the system can be stabilized using a standard proportional-derivative (PD) feedback controller

$$
\tau_{q}=\mathbf{H}\left(\mathbf{q}_{r}\right)\left(\ddot{\mathbf{q}}_{r}+\mathbf{K}_{p} \mathbf{e}+\mathbf{K}_{d} \dot{\mathbf{e}}\right)+\mathbf{h}\left(\mathbf{q}_{r}, \dot{\mathbf{q}}_{r}\right),
$$

where

$\mathbf{K}_{p}=\operatorname{diag}\left[k_{p, 1} k_{p, 2} k_{p, 3}\right], \mathbf{K}_{d}=\operatorname{diag}\left[k_{d, 1} k_{d, 2} k_{d, 3}\right](6)$

are positive definite gain matrices. Vibration compensation can be achieved by adding a term

$$
\boldsymbol{\tau}_{v}=\mathbf{J}^{T}(\mathbf{q})\left[\begin{array}{cc}
{ }_{n}^{0} \mathbf{R}(\mathbf{q}) & \mathbf{0}_{3 \times 3} \\
\mathbf{S}(\mathbf{p}){ }_{n}^{0} \mathbf{R}(\mathbf{q}) & { }_{n}^{0} \mathbf{R}(\mathbf{q})
\end{array}\right]\left[\begin{array}{l}
\mathbf{u}_{l}\left({ }^{n} \mathbf{p}^{\prime}\right) \\
\mathbf{u}_{a}\left({ }^{n} \boldsymbol{\delta}^{\prime}\right)
\end{array}\right]
$$

to the controller (5). Feedback control laws that should ensure attenuation of both linear and angular elastic displacements are denoted by the $3 \times 1$ vector functions $\mathbf{u}_{l}$ and $\mathbf{u}_{a}$. The vibration compensator (7) need to be active in the frequency range of vibrations and must not interfere with the computed torque controller. In practice, reference joint trajectories are chosen to lie below the lowest eigenfrequency of the structure. The motion controller (5) acts within the bandwidth of the joint references, while the compensator (7) acts in the frequency range of elastic vibrations. As $\tau_{q}$ and $\tau_{v}$ should act complementary to each other, we can determine them separately. The complete control law is:

$$
\tau=\tau_{q}+\tau_{v} .
$$

Design of the feedback laws $\mathbf{u}_{l}$ and $\mathbf{u}_{a}$ requires knowledge on the functions ${ }^{n} \mathbf{f}\left({ }^{n} \mathbf{p}^{\prime}\right)$ and ${ }^{n} \mathbf{m}\left({ }^{n} \delta^{\prime}\right)$. A way to identify these relations will be presented in Section 4.

\section{Experimental Set-up}

We perform our experiments on the direct-drive RRR robot shown in Fig. 2. Each joint has infinite motion range thanks to the use of sliprings for the transfer of power and sensor signals. Joint motions are measured using incremental encoders. A PC-based control system enables an easy evaluation of diverse control laws [14]. A detailed description of the robot kinematics and dynamics is available in $[15,16]$. It includes kinematic (forward and inverse) and dynamic models in closed- 
form. The dynamic model is used for implementation of the control law (5)-(8).

The direct-drive construction of the RRR robot implies a highly nonlinear and coupled robot dynamics that is quite challenging for accurate control. Joint flexibility is negligible. However, the last link is a plastic tube with a low rigidity. Its flexibility causes vibrations at the tip, complicating accurate tracking of reference tip trajectories. This link features translational elastic deflections along the $y_{3}$ and $z_{3}$ axes, indicated in Fig. 2. Deflections along $y_{3}$ are more profound, as they arise inline with movement in the last joint. Elastic deformations of the link cause translational vibrations at the tip, as high torsional stiffness of the link prevents rotational vibrations. Therefore, a sensor of translational movements is required for measuring vibrations at the tip. We adopt a triaxial accelerometer as a measuring device. This sensor has poor low frequency characteristics, as it cannot measure constant accelerations and does not provide reliable measurements up to a lower cut-off frequency of $3 \mathrm{~Hz}$. However, the vibrations arising at the tip of our robot lie within the band of the sensor's reliable measurements. We mount it at the origin of the coordinate frame ' 3 ', aligning its mutually orthogonal principal axes with the frame axes. This enables direct measurement of accelerations of translational tip vibrations.

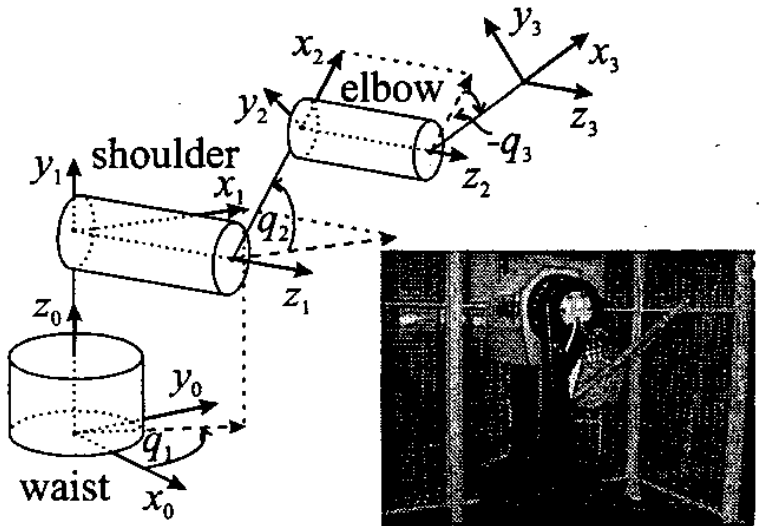

Figure 2: RRR robot experimental set-up

To illustrate the effects of link elasticity, we consider the reference joint motions shown in Fig. 3. They are realized on the robot using controller (5). In Fig. 4 we present the resulting joint velocities and accelerations. They are not measured, but reconstructed using a linear observer. The observer filters-out quantization noise caused by the incremental encoders. From the plots we see that the time-histories for the $3^{\text {rd }}$ d.o.f. reveal the presence of a high frequency component superimposed to the nominal speed and acceleration. Power spectral densities. (PSD) of $-\dot{q}_{3}$ and $\ddot{q}_{3}$, presented in Fig. 5, indicate a dominant harmonic at $28 \mathrm{~Hz}$. In the next section we will show that this harmonic corresponds to the first eigenfrequency of the last link. It turns out that the joint references shown in Fig. 3 induce elastic deflections of the last link, which influence measurements from the $3^{\text {rd }}$ position sensor.

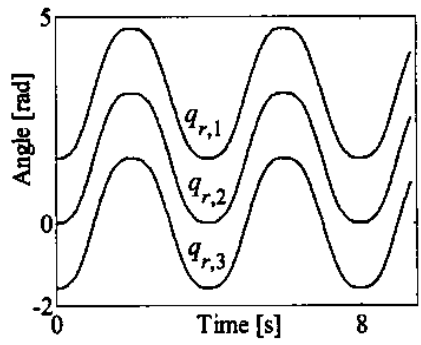

Figure 3: Reference joint motions
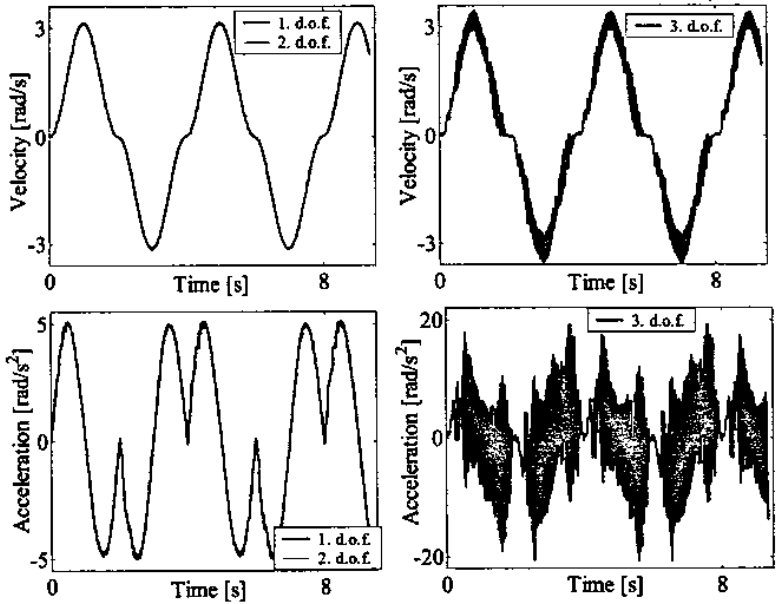

Figure 4: Resulting joint velocities and accelerations
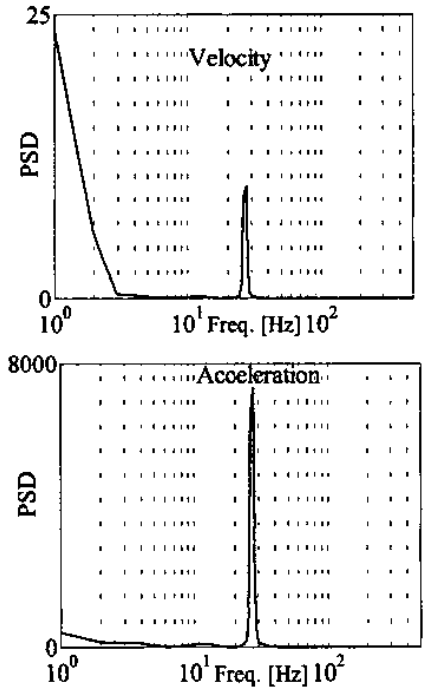

Figure 5: $P S D s^{\prime}$ of $\dot{q}_{3}$ and $\ddot{q}_{3}$

Signals from the triaxial accelerometer should confirm the presence of vibrations. As an illustration, we investigate signals from the accelerometers aligned with the $y_{3}$ and $z_{3}$ axes. They are shown in Fig. 6 together with the reference tip accelerations:

$$
\left[\begin{array}{l}
{ }^{3} \ddot{x}_{r} \\
{ }^{3} \ddot{y}_{r} \\
{ }^{3} \ddot{z}_{r}
\end{array}\right]={ }_{3}^{0} \mathbf{R}^{T}\left(\mathbf{q}_{r}\right)\left[\mathbf{J}_{u p}\left(\mathbf{q}_{r}\right) \ddot{\mathbf{q}}_{r}+\dot{\mathbf{J}}_{u p}\left(\mathbf{q}_{r}\right) \dot{\mathbf{q}}_{r}\right],
$$


where $\mathbf{J}_{u p}$ denotes the upper $(3 \times n)$ part of the manipulator geometric Jacobian $\mathbf{J}$. The right-hand side of (9) is premultiplied by ${ }_{3}^{0} \mathbf{R}^{T}(\mathbf{q})$ to represent the tip accelerations in the same coordinate frame (' 3 ') as the outputs from the triaxial accelerometer.
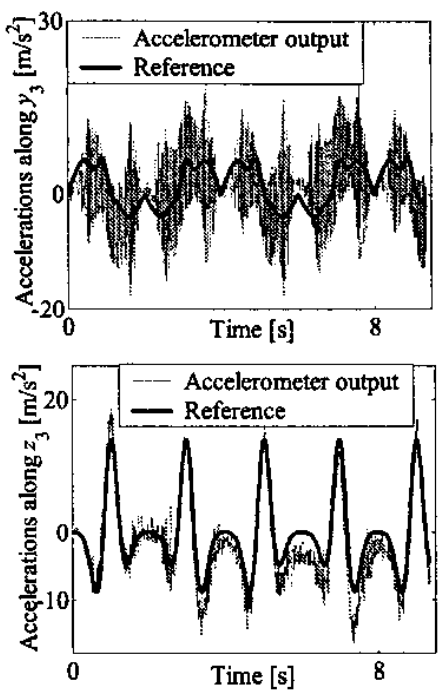

Figure 6: Reference tip accelerations and outputs from the accelerometers aligned with $y_{3}$ and $z_{3}$ axes

If we compare the expected and measured accelerations along $y_{3}$ and $z_{3}$ axes, shown in Fig. 6, we may notice the presence of a high-frequent component in the output of the accelerometer aligned with $y_{3}$. PSD of this signal, given in Fig. 7, shows that the accelerometer detects the oscillation at $28 \mathrm{~Hz}$. This frequency has already been observed in the power spectra of $\dot{q}_{3}$ and $\ddot{q}_{3}$. A good correspondence between desired and measured time histories shown on the right-hand side in Fig. 6, confirms appropriate calibration of the installed accelerometers. Slight discrepancies are due to the poor low-frequent characteristics of the sensor. The chattering in the output of the accelerometer along $z_{3}$ is not caused by oscillations, as they are negligible along this axis, but it is rather due to sensor noise and interference between the accelerometers ("cross-talk" effect).

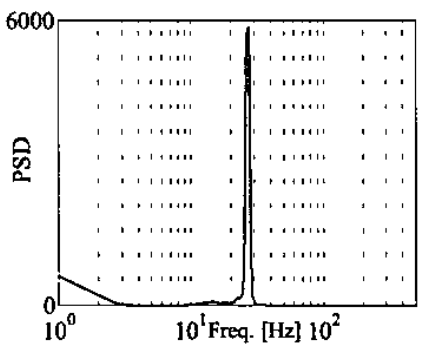

Figure 7: PSD of output from the accelerometer aligned with $y_{3}$

\section{Design of feedback vibration compensator}

In Section 2 we formulated a strategy that can remedy the problem of tip vibrations. There we stressed that designing a vibration compensator requires knowledge of a relation between the elastic force/torque and the vibration displacements/rotations. In the considered robot only translational vibrations can be found. To get the relation between the elastic force and the displacements we can either use first principles modeling [1-6] or experimental identification. The former approach is theoretically well-established, but more time-consuming. In our strategy we prefer identification, as it provides relevant models more efficiently.

Accurate information on velocity and especially position can hardly be acquired from accelerometer output signals, even if filters or observers are engaged. To avoid the need for accurate information on the linear elastic displacement of the robot tip, we directly use signals from the accelerometers, as their frequency is the same as of the vibration displacements, and their magnitudes are proportional to amplitudes of the vibrations. Strictly speaking, these relations hold for periodic single harmonic tip vibrations. However, they are practically valid even for more involved harmonic content of the vibrations [10], as the lowest eigenfrequency dominates the higher harmonics. In most practical problems it is acceptable to concentrate on the first flexible mode and to assume that displacement and acceleration of the vibrations are proportional. Smaller amplitude of the vibrations implies smaller acceleration. As the opposite also holds, instead of looking for the relation ${ }^{n} \mathbf{f}\left({ }^{n} \mathbf{p}^{\prime}\right)$ we can find a relation between ${ }^{n} \mathbf{f}$ and " $\ddot{\mathbf{p}}$, since the tip vibrations can be attenuated by counteracting the force that induces these accelerations.

Vibrations occur at specific eigenfrequencies, so we need to focus on the frequency range that covers eigenfrequencies that are interesting for us. Here, we use an $8^{\text {th }}$ order Yulewalk bandpass filter, whose Bode plot is shown in Fig. 8, to extract information about the vibrations from the accelerometer signals.

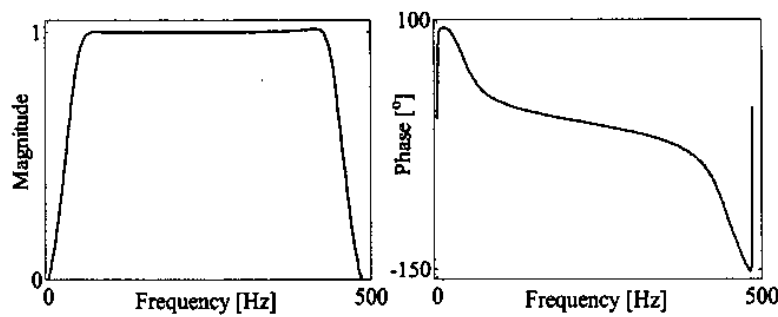

Figure 8: $8^{\text {th }}$ order Yulewalk filter used for filtering accelerometer outputs

The relation between equivalent elastic force and filtered acceleration is identified by three sets of measurements. Each set identifies the relationship ${ }^{3} f_{i}\left({ }^{3} \ddot{p}_{i}^{\prime}\right)$ $(i=x, y, z)$. By applying

$$
\tau=\tau_{q}\left(\mathbf{q}_{r}\right)+\mathbf{J}_{u p}^{T}(\mathbf{q})_{3}^{0} \mathbf{R}(\mathbf{q})^{3} \mathbf{f}(v(t)),
$$

with $\tau_{q}$ defined by (5), the robot is kept in some pres- 
cribed static configuration $\mathbf{q}_{r}\left(\dot{\mathbf{q}}_{r}=\mathbf{0}\right)$. A random noise $v(t)$ is injected into the system via ${ }^{3} f_{i}=v(t)$ $\left({ }^{3} f_{j}=0, j \neq i\right)$. Fig. 9 presents the frequency responses of the identified transfer functions ${ }^{3} \ddot{p}_{y}^{\prime}(s) /{ }^{3} f_{y}(s)$ for 16 distinct robot configurations to illustrate the effect of this excitation. The responses show consistent relation between ${ }^{3} f_{y}$ and ${ }^{3} \ddot{p}_{y}^{\prime}$, except in the low frequency region, where we could not get reliable measurements due to poor signal-to-noise ratio. Similar consistency has been observed when the transfer function ${ }^{3} \ddot{p}_{z}^{\prime}(s) /{ }^{3} f_{z}(s)$ was identified. Transfer function ${ }^{3} \ddot{p}_{x}^{\prime}(s) /{ }^{3} f_{x}(s)$ was not considered, as longitudinal vibrations of the last link (along $x_{3}$ ) are negligible. On the frequency responses presented in Fig. 9 we clearly observe two eigenfrequencies - one at $28 \mathrm{~Hz}$ and another at $180 \mathrm{~Hz}$. The first one has been already recognized as the frequency of the tip vibrations. The second one is not excited in our experiments, as it is not present in the sensor signals. Therefore, it is not further investigated. The strategy we used to identify a relation between equivalent elastic forces and linear vibration displacements could be applied also if a relation between the equivalent elastic torque and angles of rotational vibrations should be identified. Such relation, however, is not needed for the available set-up.
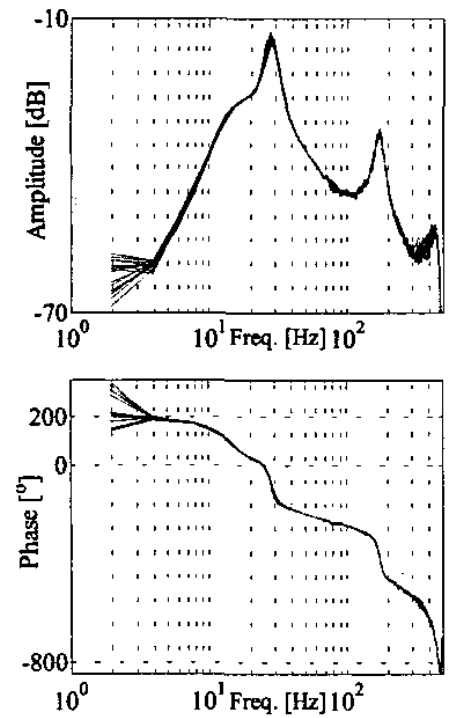

Figure 9: Identified frequency responses ${ }^{3} \ddot{p}_{y}^{\prime}(s) /{ }^{3} f_{y}(s)$

We now proceed with the design of the vibration compensator. A suitable compensator should reduce the tip vibrations at $28 \mathrm{~Hz}$, but it must not interfere with the motion controller (5), as explained in Section 2 . We do not want a contribution from the compensator within the frequency range of the reference accelerations. The vibration compensator should be active only beyond this low-frequency band, as it should act complementary to the controller of the nominal joint motions.

Here we will just discuss compensation of the vibrations along the ' $y_{3}$ ' axis. This means that we should design only the $2^{\text {nd }}$ component of the feedback compensation law $\mathbf{u}_{l}$ in (7). This law treats the tip vibrations in the same coordinate frame where the vibrations are measured (' 3 '). The objective is to keep ${ }^{3} \ddot{p}_{y}^{\prime}$ close to zero, by appropriate change of ${ }^{3} f_{y}$. It turns out that the identified transfer function ${ }^{3} \ddot{p}_{y}^{\prime}(s) /{ }^{3} f_{y}(s)$ has the role of the plant transfer function $P$, which should be feedback regulated with some compensator $C$. The vibration attenuation problem is thus simplified to a linear regulation problem, for which we have available a number of solutions (see [17] for a review). This conclusion undoubtedly simplifies the original problem of vibration compensation and represents the key contribution of the strategy we suggest in this paper. Among a number of possibilities, we employ $\mathrm{H}_{\infty}$ design for $C$. This choice naturally results from our desire to achieve robust performance of the regulated system. In turn it offers an answer to the question how to achieve robust attenuation of robot tip vibrations, which was posed in [10]. Using $\mathrm{H}_{\infty}$ theory we designed the compensator $C$. It gives the open-loop gain $P C$ which frequency response is shown in Fig. 10. The regulated system is clearly stable, its phase margin of $30^{\circ}$ is sufficient, and a high-frequency roll-off is realized. The sensitivity function $S(=1 /(1+P C)$, given in Fig. 11, shows attenuation of the first eigenfrequency of about $14 \mathrm{~dB}$, but a larger $S$ on both sides of $28 \mathrm{~Hz}$. The peaks of $S$ are below $7 \mathrm{~dB}$, which can be tolerated.

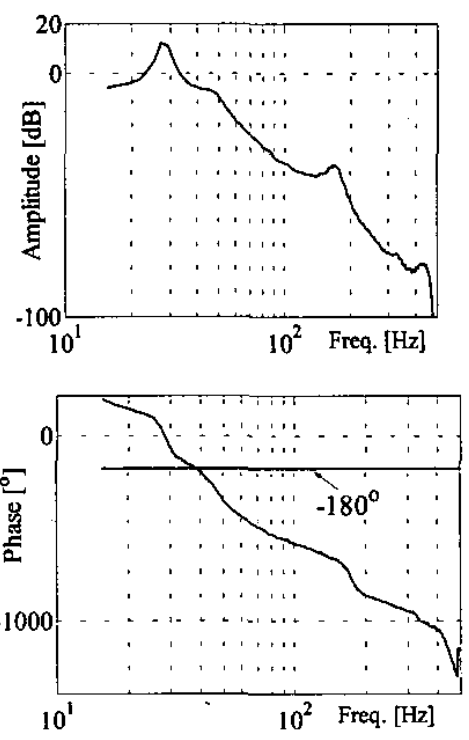

Figure 10: Open-loop gain frequency response of the regulated system 


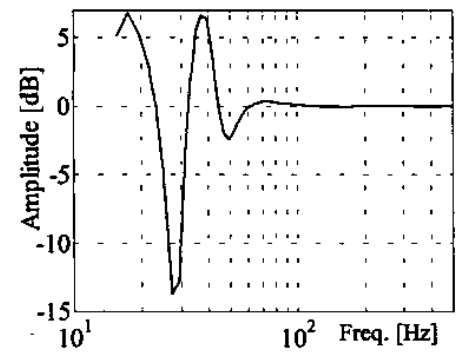

Figure 11: Sensitivity function of the regulated system

\section{Experimental evaluation}

In this section we present experimental results in reducing vibrations using the designed compensator. First we consider the motion task illustrated in Fig. 3. If the vibration compensator is disabled, the output of the accelerometer aligned with the $y_{3}$ axis is as shown on the left-hand side in Fig. 6. The accelerometer output shown on the left-hand side in Fig. 12 is obtained if the vibration compensator is enabled. Clearly, there is a significant attenuation of the vibrations in the latter case. This is also confirmed by the right side of Fig. 12, showing negligible power of the accelerometer output at $28 \mathrm{~Hz}$ if the vibration compensator is active.
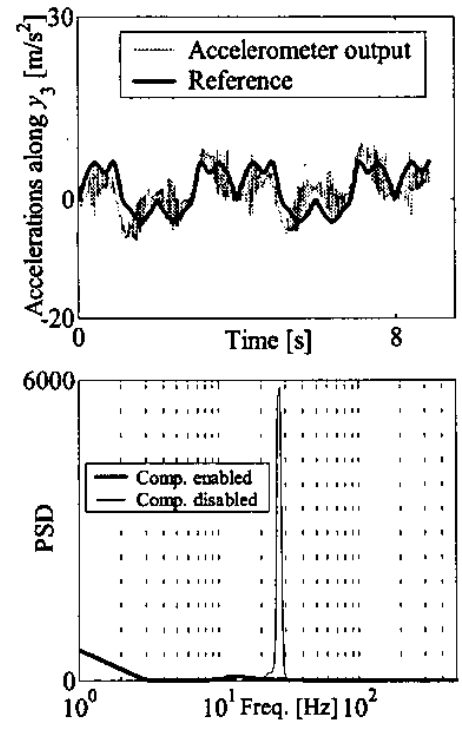

Figure 12: Left: reference and measured acceleration along $y_{3}$ with vibration compensator enabled;

right: PSDs' of the measured acceleration along $y_{3}$

To test robustness of the designed compensator, the $28 \mathrm{~Hz}$ resonance of the last link is amplified by increasing $k_{d, 3}$ gain in the motion controller (5). The frequency response ${ }^{3} \ddot{p}_{y}^{\prime}(s) /{ }^{3} f_{y}(s)$ corresponding to an increased $k_{d, 3}$ differs from the one shown in Fig. 9, which was used for the design of the vibration compensator. The difference may occur since increase of $k_{d, 3}$ amplifies high frequent eigenmodes and tilts the frequency response of $P$. The robot tip should write the letters shown on the left-hand side in Fig. 13. The letters are written in a vertical surface, parallel to the $x_{0} z_{0}$ plane. The corresponding joint motions are given next to the letters. Writing the letters demands a considerable dynamic engagement from the robot [18]. At the beginning of the experiment the vibration compensator is disabled. Execution of the reference joint motions, shown in Fig. 13, generates significant chattering in the acceleration of the $3^{\text {rd }}$ joint and in the outputs of the triaxial accelerometer. When the vibration compensator is enabled, an immediate attenuation of the vibration occurs. This is illustrated in Fig. 14, representing reference and actual accelerations of the $3^{\text {rd }}$ joint (left), and reference and measured acceleration along the $y_{3}$ axis (right). In Fig. 15 we show a photo of the executed sequence of letters. We may see that the compensator was enabled when writing the letter ' 0 '. Before enabling the compensator, the letters were written with considerable oscillations around the reference path. When enabled, the compensator attenuates the oscillations and the writing proceeds smoothly and accurately. Cartesian errors along $x_{0}$ and $z_{0}$ directions, presented in Fig. 16, reduce when the vibration compensation is activated. This example shows robustness of the compensator with respect to variations in plant dynamics.
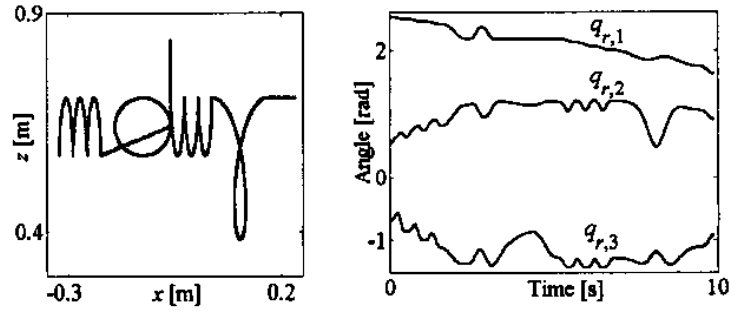

Figure 13: Left: reference path of the robot tip; right: the corresponding reference joint motions
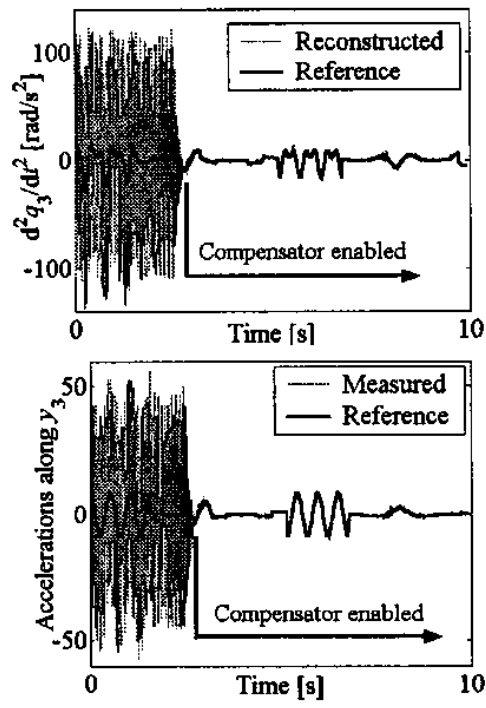

Figure 14: Left: reconstructed executed acceleration of the $3^{\text {rd }}$ joint; right: measured acceleration along $y_{3}$ 


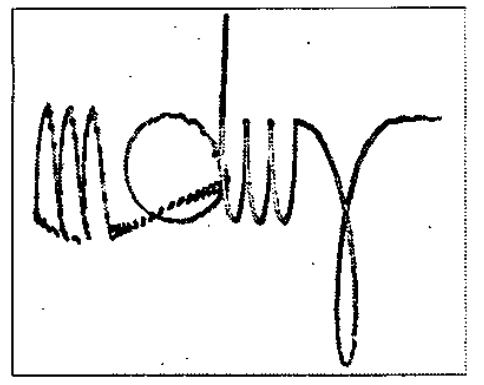

Figure 15: Photo of the path executed by the robot tip
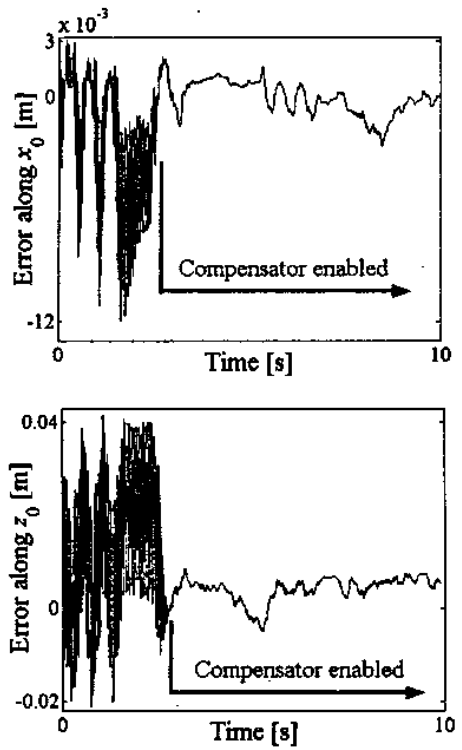

Figure 16: Cartesian errors along $x_{0}$ and $z_{0}$ directions

\section{Conclusions}

We present a method for reducing vibrations arising at the tip of a robot due to the presence of flexibility. The method requires knowledge of flexible dynamics and sensors for measuring vibrations. Vibrations are measured using accelerometers. A relevant model of elastodynamics is experimentally identified. Identification makes obtaining a verified model more efficient. Identified model is combined with a rigid-body model. The combined model enables regulation of tip vibrations directly in the coordinate frame where the vibrations are measured. The problem of vibration compensation is thus reduced to an ordinary regulation problem, solvable using a well-developed linear theory. This gives more freedom in choosing a particular form of the vibration compensator. Design of a robust vibration compensator is proposed in this paper. Practical usability and robust performance of the compensator are experimentally verified on a spatial direct-drive robot.

\section{References}

[1] M.C. Readman, Flexible Joint Robots, CRC Press, Boca Raton, Florida, 1994.

[2] V. Potkonjak, "Contribution to the Dynamics and Control of Robots Having Elastic Transmissions," Robotica, Vol. 6, No.1, 1988, pp. 63-69.

[3] W.J. Book, "Recursive Lagrangian Dynamics of Flexible Manipulator Arms," The Int. J. Rob. Res., Vol. 3, No. 3, 1984, pp. 87-101.

[4] D. Surdilović, M. Vukobratović, "One Method for Efficient Dynamic Modeling of Flexible Manipulators," Mech. and Mach. Theory, Vol. 31, No. 3, 1996, pp. 297-315.

[5] I. Kruise, Modelling and Control of a Flexible Beam and Robot Arm, PhD Thesis, University of Twente, Enschede, The Netherlands, 1990.

[6] A. De Luca, "Feedforward/Feedback Laws for the Control of Flexible Robots," Proc. IEEE Int. Conf. Rob. Autom., San Francisco, California, April 2000, pp. 233-240.

[7] D.M. Gorinevski, A.V. Lensky, E.I. Savbitov, "Feedback Control of a One-link Flexible Manipulator With a Gear Train," J. Rob. Sys., Vol. 8, No. 5, 1991, pp. 659-676.

[8] Z.H. Luo, D.X. Feng, "Nonlinear Torque Control of a Single-Link Flexible Robot," J. Rob. Sys., Vol. 16, No. 1, 1999, pp. 25-35.

[9] S. Yurkovich, A.P. Tzes, I. Lee, K.L. Hillsley, "Control and System Identification of a Two-Link Flexible Manipulator," Proc. IEEE Int. Conf. Rob. Autom., Cincinnati, Ohio, May 1990, pp. 1626-1631.

[10] F. Matsuno, Y. Sakawa, "A Simple Model of Flexible Manipulators with Six Axes and Vibration Control by Using Accelerometers," J. Rob. Sys., Vol. 7, No. 4, 1990, pp. 575-597.

[11] S. López-Linares, A. Konno, M. Uchiyama, "Vibration Suppression Control of 3D Flexible Robots Using Velocity Inputs,"J. Rob. Sys., Vol. 14, No. 12, 1997, pp. 823-837.

[12] M. Vukobratović, V. Potkonjak, Dynamics of Manipulation Robots: Theory and Application, Springer-Verlag, Berlin, 1982.

[13] L. Sciavicco, B. Siciliano, Modeling and Control of Robot Manipulators, McGraw-Hill, Singapore, 1996.

[14] B. van Beek, B. de Jager, "An Experimental Facility for Nonlinear Robot Control," in Proc. IEEE Int. Conf. Control Appl., Hawai'i, USA, 1999, pp. 668-673.

[15] D. Kostić, R. Hensen, B. de Jager, and M. Steinbuch, "Closed-form Kinematic and Dynamic Models of an Industrial-like RRR Robot," IEEE Int. Conf. Rob. Autom., Washington D.C., May 2002, pp. 1309-1314.

[16] D. Kostić, R. Hensen, B. de Jager, and M. Steinbuch, "Modeling and Identification of an RRR-robot," IEEE Conf. Dec. Control, Oriando, Florida, December 2001, pp. 1144-1149.

[17] M. Steinbuch, M.L. Norg, "Advanced Motion Control," Europ. J. of Control, Vol. 4, No. 4, 1998, pp. 278-293.

[18] V. Potkonjak, S. Tzafestas, D. Kostic, "Concerning the Primary and Secondary Objectives in Robot Task Definition-The "Learn From Humans" Principle," Math. and Comp. in Simul., Vol. 54, No. 1-3, 2000, pp. 145-157. 